\title{
Exchanging More Than Complete Data
}

\author{
Marcelo Arenas \\ Department of Computer Science \\ Pontificia Universidad Católica de Chile \\ marenas@ing.puc.cl
}

\begin{abstract}
In the traditional data exchange setting source instances are restricted to be complete, in the sense that every fact is either true or false in these instances. Although natural for a typical database translation scenario, this restriction is gradually becoming an impediment to the development of a wide range of applications that need to exchange objects that admit several interpretations. In particular, we are motivated by two specific applications that go beyond the usual data exchange scenario: exchanging incomplete information and exchanging knowledge bases.

In this talk, we propose a general framework for data exchange that can deal with these two applications. More specifically, we address the problem of exchanging information given by representation systems, which are essentially finite descriptions of (possibly infinite) sets of complete instances, and then we show the robustness of our proposal by applying it to the problems of exchanging incomplete information and exchanging knowledge bases, which are both instantiations of the exchanging problem for representation systems.
\end{abstract}

